\title{
Investigation of the effect of donor-acceptor substitution on band gap, band width, and conductivity
}

\author{
U. Salzner* \\ Department of Chemistry, Bilkent University, 06533 Bilkent, Ankara, Turkey
}

\begin{abstract}
Polymers of two donor-acceptor systems, 3-cyano,3'-hydroxybithiophene 1 and 4-dicyanomethylene-4H-cyclopenta[2,1-b:3,4-b'],3,4ethylenedioxythiophene (CDM-EDOT) 2, were analyzed with density functional theory. As predicted by perturbation theory, interactions between donors and acceptors with very different energy levels are greatly reduced compared to those between fragments with similar energy levels. This leads to localized states and bands with little dispersion. For poly-1 these localized states lie below the valence band and above the conduction band. For 2 localized unoccupied levels lie within the band gap. These acceptor levels account for the high electron affinity of poly-2 and allow for self-doping. Self doping explains the increased intrinsic conductivity of poly-2, the localized nature of the low lying MOs rationalizes the low mobility of n-type carriers in poly-2.
\end{abstract}

Keywords: Density functional calculations, donor-acceptor concept, band gap, band width, conductivity

\section{Introduction}

The donor-acceptor concept[1] predicts that polymers with repeat units composed of a donor with high lying energy levels and an acceptor with low lying energy levels have small band gaps and wide valence and conduction bands. The underlying idea is that the polymer valence band forms energetically near the HOMO of the donor and that the conduction forms in the region of the LUMO of the acceptor. Considering perturbation theory, this expectation appears rather strange. Band formation requires strong interaction between the fragments and this interaction is inversely proportional to the energy difference between the fragment orbitals. Therefore donor-acceptor substitution should hamper band formation. Numerous donor-acceptor polymers were synthesized and experimentally investigated. In several studies success of the donor-acceptor concept has been claimed. Here theoretical electronic structure analysis of poly-1 and poly-2 is presented and the effect of donor-acceptor on electrical properties in analyzed.

\section{Methods}

Oligomers of 1 and 2 were optimized using density functional theory (DFT). Becke's three parameter hybrid functional was combined with Perdew and Wang's correlation functional. The weight of the Hartree-Fock exchange was raised to $30 \%$. Stevens Basch Krauss pseudo-potentials and split valence plus polarization basis sets were employed.[2] The backbones are planar although the ethylenedioxy group is twisted. Orbital contour plots were done with gopenmol. All calculations were performed with Gaussian 98.

*Ulrike Salzner. Tel: +90-312-290-2122; fax: +90-290-312-

2122; E-mail: salzner@fen.bilkent.edu.tr

\section{Results and Discussion}

In Figure 1 an orbital correlation diagram is shown for the interaction between hydroxy- and cyanothiophene. The repeat unit of 1 has a $0.21 \mathrm{eV}$ higher lying HOMO than hydroxy-thiophene and a $0.33 \mathrm{eV}$ lower lying LUMO than cyano-thiophene. In contrast, dimerization of hydroxythiophene leads to an increase in HOMO energy of $1.03 \mathrm{eV}$ and dimerization of cyanothiophene to a decrease in LUMO energy of $1.44 \mathrm{eV}$. The HOMO-LUMO gap of the mixed dimer is only $0.20 \mathrm{eV}$ smaller than that of bithiophene.

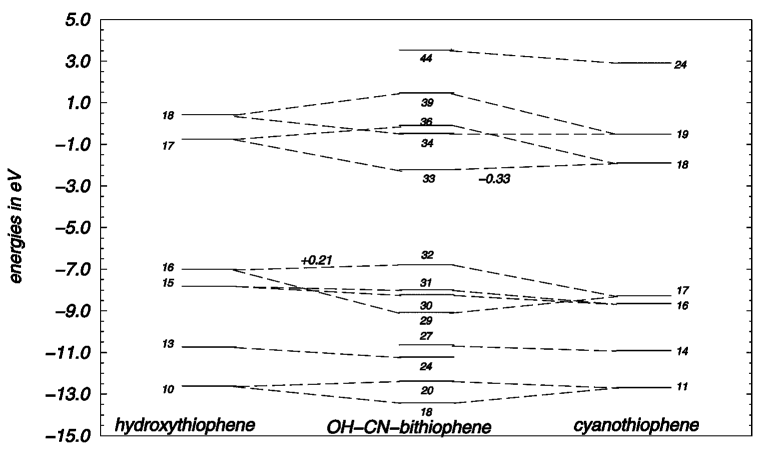

Fig. 1 Energy levels of hydroxythiophene, cyanothiophene, and their mixed dimer 
HOMO and LUMO of the mixed dimer are very similar in character and energy to those of bithiophene. The extrapolated band gap, obtained by using monomer through tetramer, is 0.03 $\mathrm{eV}$ larger than that of polybithiophene. Valence and conduction bands are delocalized and show slightly smaller dispersions than those of polythiophene. HOMO and LUMO of the trimer (Fig. 2) look similar to those of sexithiophene. Localization occurs in lower and higher lying levels. Two low lying localized MOs are shown in Fig.2. In agreement with experiment[3] properties of 1 are average between those of the homopolymers.
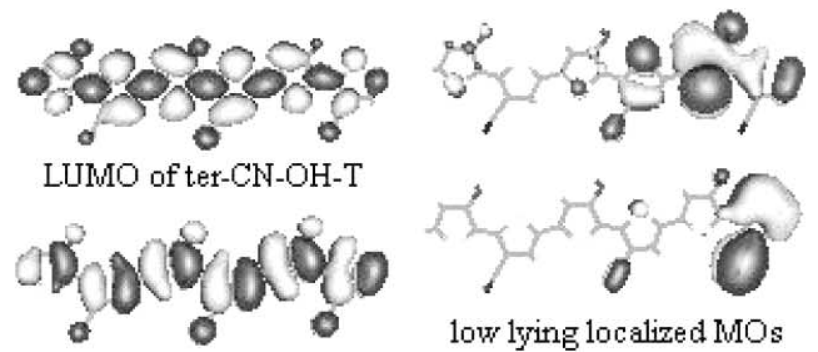

low lying localized MOs

HOMO of ter-CN-OH-T of ter-CH-OH-T

Fig. 2 MOs for ter-1

Poly-2 has a smaller band gap $(1.63 \mathrm{eV})$ than poly-EDOT $(2.11$ $\mathrm{eV})$ or poly CDM $(1.72 \mathrm{eV})$. This has been attributed to donoracceptor substitution. [4] Fig. 3 shows the relationship between energy levels in thiophene, EDOT, bithiophene, CDM, and 2. The HOMO of EDOT is shifted upwards compared to thiophene. The HOMO of bithiophene lies much higher than that of thiophene. The dicyanomethyene lowers the HOMO energy so that CDM and EDOT have about the same IP. Joining EDOT and CDM allows for interaction of orbitals with similar energies and leads to a large splitting. There is no donor-acceptor character in the HOMO of 2. Upon polymerization a valence band (compare HOMO of ter-2 in Fig. 4) forms that is similar in energy and dispersion to that of polybithiophene. The LUMO energies of EDOT and PCDM differ by $3.4 \mathrm{eV}$. As a result these orbitals do not interact and the LUMO of 2 is localized at CDM. Upon polymerization no band formation occurs (compare Figs. 4 and 5). The EA of poly-2 is with $3.98 \mathrm{eV}$ practically identical to that of the CDM monomer $(3.94 \mathrm{eV})$. In contrast, the LUMO of EDOT interacts stronly with $\mathrm{LUMO}+1$ and LUMO +2 of CDM.

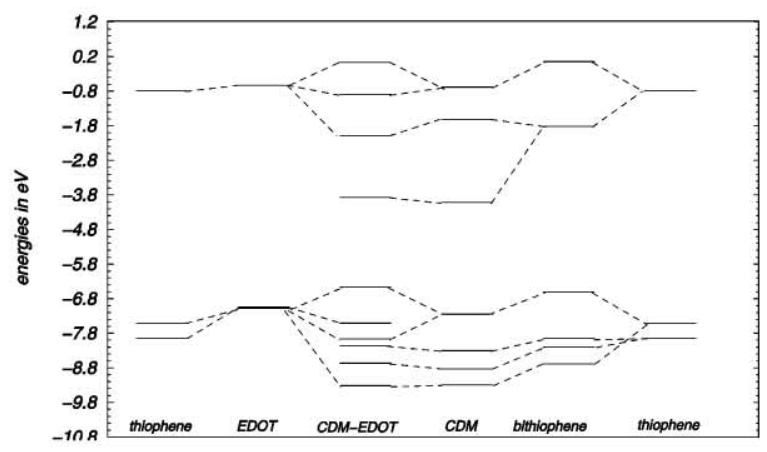

Fig. 3 Energy levels for thiophene, bithiophene, EDOT, CDM, and the repeat unit of 2 .

The resulting band is similar in character to the conduction band of polybithiophene. Thus, in the conduction band region strong donor-acceptor character of the interaction fragments leads to

localized acceptor levels within the band gap. Experimentally a very small band gap was found for poly-2 but the mobility of $n$ type carriers was 500 times smaller than that of p-type carriers. [4] The localized nature of the conduction "band" accounts for this observation.

Fig. 4 HOMO, LUMO and intergap level of the EDOT-pCDM

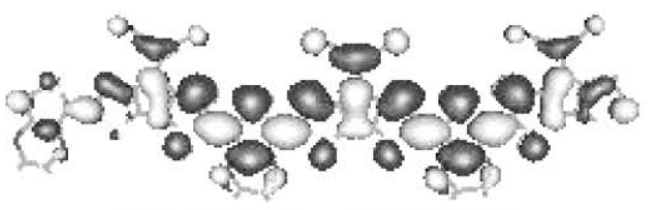

LUMO+2 of ter-CDM-EDOT

beginning band formation

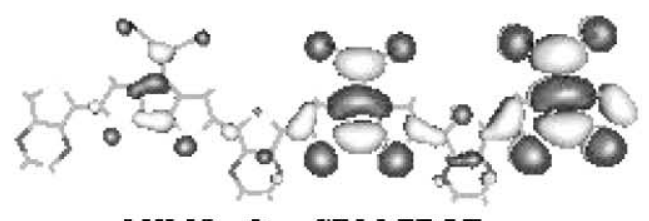

LUMO of ter-CDM-EDOT

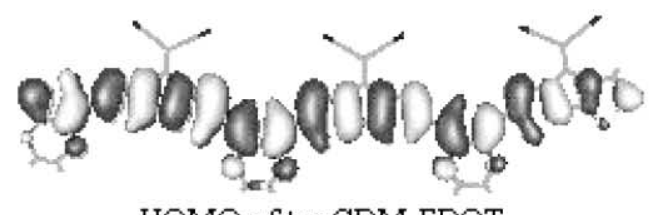

HOMO of ter-CDM-EDOT

trimer.

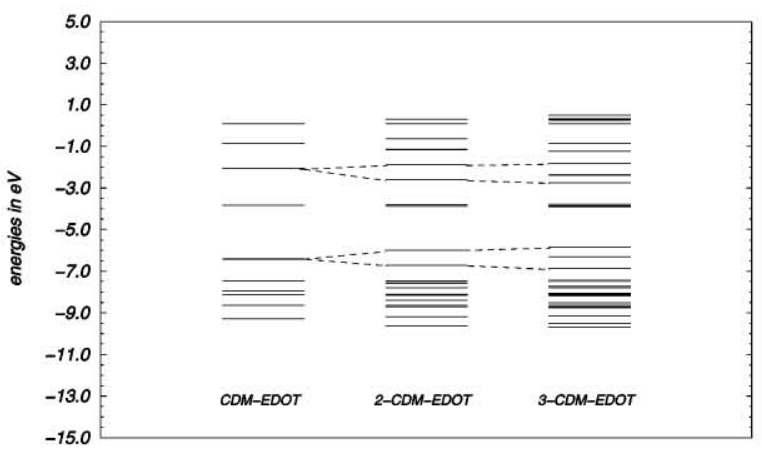

Fig. 5 Formation of bands from monomer to trimer of poly-2

\section{Conclusions}

As predicted by perturbation theory, donor-acceptor substitution results in reduced interaction between the fragments and has a negative effect on band widths and band gap decrease with increasing chain lengths. The small band gap of poly-2 is due to the dispersion of the valence band which has no donor-acceptor character and the presence of low lying localized acceptor levels. This polymer confirms that donor-acceptor substitution can not lead to small band gaps and wide bands at the same time.

[1] E. E. Havinga, W. ten Hoeve, H. Wynberg, Synth. Met. (1993) 55-57, 299.

[2] Æ. Frisch, M. J. Frisch, Gaussian 98 User's Reference, $2^{\text {nd }}$ ed., Gaussian, Inc.: Pittsburgh, 1994-1999.

[3] F. Demanze, A. Yassar, F. Garnier, Macromol. (1996) 29, 4267.

[4] H. Huang, P. G. Pickup, Chem. Mater. (1998) 8, 221. 